\section{IDDF2019-ABS-0103 THE VALUE OF THE BISAP SCORE FOR PREDICTING THE SEVERITY OF ACUTE PANCREATITIS IN PATIENTS ACCORDING TO THE 2012 REVISED ATLANTA CLASSIFICATION}

Le Thi Thu Hien*. Thai Nguyen University of Medicine and Pharmacy, Vietnam

\subsection{6/gutjnl-2019-IDDFabstracts. 146}

Background Severe acute pancreatitis (SAP) has high mortality and its early identification is important for management and risk stratification. The bedside index for severity in acute pancreatitis (BISAP) is a simple scoring system within the first 24 hours of admission which predicts the severity of pancreatitis. There were no studies designed to validate this system according to the 2012 revised Atlanta classification in Vietnam and more data are needed before clinical practice.

Aims To evaluate the BISAP score in prognostication of acute pancreatitis (AP).

Methods A prospective observational study of 68 patients presenting with AP in Thai Nguyen National Hospital from May 2018 to February 2019 were included in the study. All the patients were calculated and stratified according to the BISAP score within the first 24 hours of admission. Computerized abdominal scans are used for diagnosis and evaluation of patients with AP. SAP was defined as the persistence of organ failure for more than $48 \mathrm{~h}$. The capacity of the BISAP to predict severity, pancreatic necrosis and organ failure were evaluated using linear-by-linear association. The predictive accuracy of the BISAP score was measured as the area under the receiver operating characteristic curve (AUC).

Results SAP accounted for 20.58\%. Pancreatic necrosis accounted for $21.23 \%$. Organ failure accounted for $20.58 \%$. A BISAP score of $\geq 3$ was a statistically significant cutoff value. Risks of SAP were increased among group of BISAP $\geq$ 3 (OR: 51.56; 95\% confidence interval (CI): 3.23 - 698.56). BISAP in predicting SAP (AUC: 0.823; 95\% CI: 0.71 0.992) and organ failure (AUC: 0.871 ; 95\% CI: 0.73 0.921). BISAP $\geq 3$ predicted SAP with sensitivity: $69.65 \%$, specificity: $97.89 \%$, positive predictive values: $82 \%$, negative predictive values: $96.3 \%$.

Conclusions The BISAP predicts severity and organ failure in AP very well. BISAP score may be a valuable tool for risk stratification and prognostic prediction in Vietnamese patients with AP.

Acute pancreatitis (AP); BISAP score (Bedside index of severity in acute pancreatitis score).

\section{IDDF2019-ABS-0104 GASTROINTESTINAL CYTOMEGALOVIRAL INFECTION IN IMMUNOCOMPETENT PATIENTS IS ASSOCIATED WITH MORE SEVERE ILLNESSES AND HAS A HIGHER MORTALITY RATE COMPARED TO INFECTION IN IMMUNOCOMPROMISED PATIENTS}

Thanaboon Chaemsupaphan*, Julajak Limsrivilai, Nonthalee Pausawasdi, Phunchai Charatcharoenwitthaya, Aswin Sudcharoen. Division of Gastroenterology, Department of Medicine, Faculty of Medicine Siriraj Hospital, Mahidol University, Thailand

10.1136/gutjnl-2019-IDDFabstracts. 147
Background Gastrointestinal (GI) cytomegaloviral (CMV) infection is common among patients with immunocompromised (ICM) status. There have been some case reports in immunocompetent (ICT) patients, but the data are limited.

Methods A retrospective study of all patients diagnosed with CMV GI disease by demonstration of CMV viral inclusion or immunohistochemistry on tissue pathology from 2008 to 2017 in a tertiary referral center was performed. Immunocompromised patients included patients with AIDS, organ transplantation, or received chemotherapy, systemic corticosteroids, or immunosuppressive agents. The baseline characteristics, presentation, comorbid conditions, treatment, and outcomes were compared between the patients with ICT and ICM status.

Results 173 patients comprising 56 ICT and 117 ICM patients were included. ICT patients were older than ICM patients with a mean age of 73 and 48.6 years, respectively $(\mathrm{p}<.0001) .50 \%$ of ICT patients developed the disease during hospitalization while it was $35.9 \%$ in the ICM patients $(\mathrm{p}=0.07)$. Duration of presenting symptoms was shorter in ICTs compared to ICMs with duration of 1 and 10 days, respectively $(\mathrm{p}=0.002)$. Gl bleeding was the leading presentation in the ICT patients while diarrhea and abdominal pain were found more in the ICM patients. Extraintestinal CMV manifestation was not observed in ICT, but was found in 8 $(6.8 \%)$ in the ICM $(p=0.05)$. CMV viral load was negative in $40.7 \%$ of ICT patients compared to $12.9 \%$ in ICM patients $(p=0.002)$. The ICT patients needed more ventilator support $(37.5 \%$ in ICT and $18 \%$ in ICM, $p=0.005)$ and inotropic agents $(21.4 \%$ and $5.1 \%$ in ICT and ICM respectively, $\mathrm{p}=0.001)$. Gangcyclovir was the main treatment in both groups. $15 \%$ of ICT patients required surgery whereas $7 \%$ in ICM patients $(p=0.087)$. Mortality was significantly higher among the ICT patients with a rate of $42 \%$ compare to $23.6 \%$ in ICM patients $(p=0.018)$.

Conclusions CMV GI disease in ICT patients was frequently developed in elderly and half-developed while hospitalization. Gastrointestinal bleeding was the most common presentation. The CMV disease in ICT patients was associated with more severe illnesses and a higher mortality rate. CMV viral load was not helpful in diagnosis.

\section{IDDF2019-ABS-0105 SYSTEMATIC REVIEW WITH META- ANALYSIS: PARTIAL ENTERAL NUTRITION FOR MAINTENANCE OF REMISSION IN CROHN'S DISEASE}

Hongsheng Yang*, Yun Qiu, Rui Feng, Shu Xu, Xiuxue Hao, Shenghong Zhang, Minhu Chen. Department of Gastroenterology, The First Affiliated Hospital, Sun Yat-sen University, China

\subsection{6/gutjnl-2019-IDDFabstracts. 148}

Background Extensive enteral nutrition, which exhibits therapeutic efficacy for induction of remission, is distinguished by having a good safety profile and improvements to growth parameters. However, it remains unclear whether partial enteral nutrition, i.e., enteral nutrition along with a daily diet, is as effective as exclusive enteral nutrition in maintaining remission of Crohn's disease (CD). We aim to evaluate the efficacy and safety of partial enteral nutrition for the 
maintenance of remission in $\mathrm{CD}$ by performing a systematic review and meta-analysis.

Methods Electronic databases were searched for prospective controlled studies evaluating partial enteral nutrition for maintenance of remission in CD. The primary outcome was clinical relapse as defined by the primary studies.

Results Three randomized controlled trials and five nonrandomized controlled trials were included. The rate of clinical relapse, within a follow-up of $0.5-2$ years, was significantly lower in $\mathrm{CD}$ patients receiving partial enteral nutrition $(420$ to $1800 \mathrm{kcal} / \mathrm{d})$ than in those not receiving nutrition therapy (RR 0.58, 95\% CI, 0.45-0.75, P<0.01; NNT = 5, $P<0.01 ; \mathrm{n}=429$ ). Among $\mathrm{CD}$ patients, those receiving partial enteral nutrition exhibited a higher frequency of maintaining clinical remission (defined as CDAI <150) at 1 year $(63 \%, 89 / 141)$ than did those not receiving nutrition therapy (42\%, 52/125; RR 1.47, 95\% CI, 1.17-1.85, $P<0.01$ ). Adverse events caused by partial enteral nutrition were rare $(7 \%, 9 / 131)$ and mild.

Conclusions Partial enteral nutrition appears to be more effective than the absence of enteral nutrition therapy for maintenance of remission in $\mathrm{CD}$ and has a good safety profile.

\section{IDDF2019-ABS-0109 ULTRASOUND ELASTOGRAPHY FOR THE DETECTION OF FIBROTIC BOWEL STRICTURES IN PATIENTS WITH CROHN'S DISEASE: A SYSTEMATIC REVIEW AND META-ANALYSIS}

${ }^{1}$ Giovanni Marasco*, 'Amanda Vestito, ${ }^{2}$ Giovanni Maconi, 'Davide Festi, ${ }^{1}$ Franco Bazzoli, ${ }^{1}$ Rocco Maurizio Zagari. 'University of Bologna, Italy; ${ }^{2}$ University of Milan, Italy

\subsection{6/gutjnl-2019-IDDFabstracts. 149}

Background Ultrasound (US) elastography has been proposed as a tool for the non-invasive diagnosis of bowel fibrotic stricture in patients with Crohn's disease (CD). However, the diagnostic value of US elastography is still unclear. The aim of the study is to assess the diagnostic value of abdominal US elastography in detecting fibrotic bowel strictures in patients with CD.

Methods MEDLINE via the PubMed, Ovid Embase, Scopus and Cochrane Library databases, and abstracts of international conference proceedings were searched up to 31st March 2018. Studies were included if they assessed the performance of abdominal US elastography in detecting fibrotic bowel strictures in patients with CD using histology or the need for surgery after medical treatment as a reference standard. The quality of the studies was assessed using QUADAS-2 (Quality Assessment of Diagnostic Accuracy Studies).

Results Six studies including a total of 217 patients with CD and 231 bowel segments were selected. Three studies used strain ratio and three studies used strain value as parameters of bowel stiffness. Both the pooled mean strain ratio and the pooled mean strain value were higher in bowel segments with fibrotic strictures than in those without fibrotic strictures with a pooled standardized mean difference of 0.85 (95\% confidence level [CI]: 0 to $1.71 ; \mathrm{p}=0.05)$ (figure $1 \mathrm{~A}$ ) and 1.0 (95\% CI: -0.11 to $2.10 ; \mathrm{p}=0.08$ ) (figure $1 \mathrm{~B})$, respectively. There was a high heterogeneity between studies and all studies were at "high risk" or "unclear risk" of bias.

Conclusions This study provides evidence, albeit not robust, that US elastography could be able to detect fibrotic bowel strictures in patients with $\mathrm{CD}$ and could play a relevant role in the management of $\mathrm{CD}$ patients. Well-designed high-quality studies to assess the sensitivity and specificity of US elastography in the diagnosis of fibrotic bowel strictures in patients with CD should be implemented.

\section{IDDF2019-ABS-0110 A PREDICTIVE MODEL IDENTIFIES PATIENTS LESS LIKELY TO HAVE ADENOMAS AFTER A COLON CANCER}

${ }^{1}$ Leonardo Frazzoni* ${ }^{*}{ }^{1}$ Michele Scagliarini, ${ }^{1}$ Liboria Laterza, ${ }^{2}$ Cristina Trovato, ${ }^{3}$ Alessandro Mussetto, ${ }^{4}$ Mario De Bellis, ${ }^{5}$ Silvia Paggi, ${ }^{6}$ Cristiano Spada, ${ }^{1}$ Laura Petrella, ${ }^{1}$ Veronica Smania, ${ }^{1}$ Franco Bazzoli, ${ }^{1}$ Luigi Ricciardiello, ${ }^{5}$ Franco Radaelli, ${ }^{7}$ Cesare Hassan, ${ }^{1}$ Lorenzo Fuccio. ${ }^{1}$ University of Bologna, Italy; ${ }^{2}$ European Institute of Oncology, Milan, Italy; ${ }^{3}$ S. Maria delle Croci Hospital, Ravenna, Italy; ${ }^{4}$ University of Verona, Italy; ${ }^{5}$ Valduce Hospital, Como, Italy; ${ }^{6}$ Fondazione Poliambulanza, Brescia, Italy; ${ }^{7}$ Nuovo Regina Margherita Hospital, Rome, Italy

\subsection{6/gutjnl-2019-IDDFabstracts. 150}

Background Patients with prior colorectal cancer (CRC) are at slightly increased risk of metachronous colorectal neoplasms, therefore endoscopic surveillance is indicated. Current recommendations of repeating examinations at 1,3 and 5 years after surgery, are not tailored according to risk stratification. Our aim was to find predictive factors of colorectal neoplasms to build a predictive model, to spare colonoscopies for lowrisk patients.
B

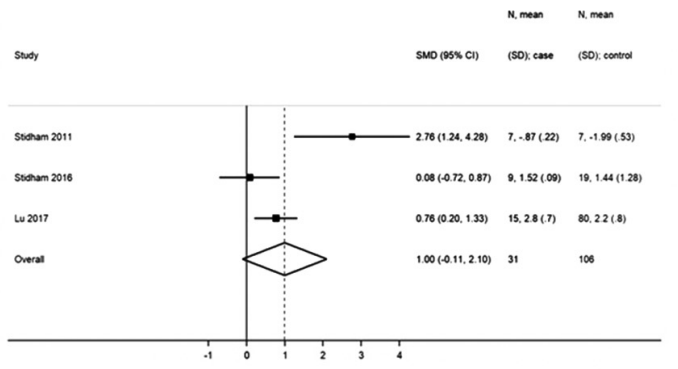

